\title{
Correction to: Algorithms for Discrete Areal Feature Generalization
}

\section{Correction to:}

Chapter 7 in: H. Yan, Description Approaches and Automated Generalization Algorithms for Groups of Map Objects, https://doi. org/10.1007/978-981-13-3678-2_7

The original version of this book was inadvertently published without citations to "Cetinkaya S., Basaraner M., Burghardt D., 2015, Proximity-based grouping of buildings in urban blocks: a comparison of four algorithms, Geocarto International, 30(6): 618-632".

The correct citation has been included as reference citation in text on page 223 and the reference has been listed on page 225 . 